\title{
Estudo de viabilidade do aproveitamento energético do gás de aterro na Cidade de Juiz de Fora (Minas Gerais, Brasil) considerando três cenários diferentes
}

\section{Jonhson Herlich Roslee Mensah*, Carla Sonaria Rita}

Mestrado em Engenharia de Energia. Universidade Federal de Itajubá (UNIFEI). Avenida BPS, 1303. Pinheirinho, Itajubá-MG, Brasil (CEP 37500-903). *E-mail: mjherlich@gmail.com.

Resumo. Impactos ambientais negativos tais que a poluição ambiental, o aquecimento global, a degradação do solo e lençol freático e também as doenças relacionadas são consequências das ações antrópicas realizadas pelo ser humano. Note-se que a base de todos esses problemas ambientais é a geração de resíduos sólidos que está diretamente relacionada ao crescimento populacional. Assim, tornou-se importante o estudo desses resíduos gerados para ter um conhecimento sobre suas composições e classifica-las além de trata-las e dar um destino final adequado a eles a fim de garantir uma qualidade de vida melhor para as gerações futuras. Diante desse fato, surgiu a Política Nacional de Resíduos Sólidos (PNRS), que descreveu a necessidade de limpeza urbana e coleta seletiva, a partir da implantação de metas para melhor destinação desses resíduos. Para ter uma noção da quantidade de resíduos sólidos gerada na cidade de Juiz de Fora (MG), realizou-se uma projeção populacional para um período de 20 anos, seguida de uma análise da construção de um aterro sanitário para o mesmo período de projeto. Em seguida, através do programa Waste Reduction Model (WARM) foi feita a análise de três cenários, considerando a reciclagem de materiais e uso para compostagem, sendo os cenários 2 e 3 com o aproveitamento de gás para geração energética. De posse dos resultados obtidos para cada cenário, realizou-se a análise comparativa da viabilidade econômica deles e por fim determinou-se o melhor cenário a ser adotado para a cidade de Juiz de Fora (MG).

Palavras-chave: Aproveitamento energético; Resíduos sólidos; Coleta seletiva; Sustentabilidade.

Abstract. Feasibility study of energy use of landfill gas in the City of Juiz de Fora (Minas Gerais, Brazil) considering three different scenarios. Negative environmental impacts such as environmental pollution, global warming, soil degradation and water table and related diseases are also consequences of anthropogenic actions performed by the human. It should be
Recebido:

23/08/2018

Aceito:

$30 / 11 / 2018$

Publicado:

$31 / 12 / 2018$

Acesso aberto

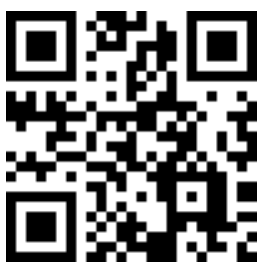

ORCID

(1) 0000-0003-0897-892X

Johnson Herlich

Roslee Mensah

D 0000-0002-9515-5456

Carla Sonaria Rita 
noted that the basis of all these environmental problems is the generation of solid waste that is directly related to the population growth. Thus, it became important to the study of this waste generated to have a knowledge of his compositions and sorts them in addition to treats them and give a final destination appropriate to them in order to ensure a better quality of life for future generations. Given this fact, did the Brazilian Solid Waste Policy (PNRS), described the need for urban cleaning and garbage collection, from the implementation of goals for best destination of such waste. To get a sense of the amount of solid waste generated in the City of Juiz de Fora (MG), was held a population projection for a period of 20 years, followed by an analysis of the construction of a landfill for the same period. Then, through the Waste Reduction Model (WARM) was made the analysis of three scenarios, considering the materials recycling and use for composting, with the 2 and 3 scenarios with the use of gas for energy generation. Based on the results obtained for each scenario, a comparative analysis economic viability of their was carried out and, finally, it was determined the best scenario to be adopted for the City of Juiz de Fora (MG).

Keywords: Energy recovery; Solid wastes; Selective collec; Sustainability.

\section{Introdução}

0 meio ambiente tem sofrido várias mudanças devido aos efeitos negativos causados pelo crescimento populacional e o desenvolvimento industrial observado nos últimos anos, tais como a poluição, a degradação dos recursos naturais e sobretudo a geração dos resíduos sólidos, que por sua vez se definem como partes de resíduos que são gerados após a produção, uso ou transformação de bens de consumos, como por exemplo os computadores, automóveis, televisores, aparelhos celulares, eletrodomésticos, etc.

De acordo com a definição dada pela norma da Associação Brasileira de Normas Técnicas (ABNT) NBR 10004:2004 (ABNT, 2004), grande parte destes resíduos pode estar nos estados sólido e semi-sólido, e é produzida nos grandes centros urbanos, principalmente, de residências, escolas, indústrias, construção civil, agrícola, hospitalar, de serviços e de varrição. Muitos destes resíduos sólidos são compostos de materiais recicláveis que são classifi- cados das seguintes maneiras: matéria orgânica (restos de comidas); papel e papelão (jornais, revistas); plásticos (garrafas, embalagens, garrafões); vidro (copos, frascos); metais (latas) e outros (roupas, óleos, resíduos eletrodomésticos), e podem retornar a cadeia de produção, gerando renda para trabalhadores e lucro para empresas. Para que isto ocorra, é necessário que haja nas cidades um bom sistema de coleta seletiva e reciclagem de lixo. Cidades que não praticam este tipo de processo, jogando todo tipo de resíduo sólido em aterros sanitários, acabam poluindo o meio ambiente. Isto ocorre, pois, muitos resíduos sólidos levam décadas ou até séculos para serem decompostos. A composição varia de população para população, dependendo da situação socioeconômica e das condições e hábitos de vida de cada um. Há que se ressaltar que conforme a referida norma ABNT, esses resíduos sólidos podem ser classificados em: Classe I (perigosos), IIA (não-inertes) e IIB (inertes). 
Apesar de ter 80\% da sua população em áreas urbanas, percebe-se que a infraestrutura e os serviços básicos não acompanham o ritmo de crescimento dos centros urbanos no Brasil (Mansor et al., 2010). Portanto, um dos serviços pendentes é o de limpeza urbana e de manejo de resíduos sólidos, e esse déficit tem um grande impacto tanto na qualidade de vida da população nos centros urbanos quanto nas áreas rurais e periféricas.

Com o surgimento da Lei $\mathrm{n}^{0}$ $12.305 / 2010$ que institui a Política Nacional de Resíduos Sólidos (PNRS), cujo objetivo principal é prever e reduzir a geração de resíduos, através da prática de hábitos de consumo sustentável, propiciar o aumento da reciclagem e a reutilização dos resíduos sólidos (aquilo que tem valor econômico e pode ser reciclado ou reaproveitado) e também o destino ambientalmente adequado dos rejeitos (aquilo que não pode ser reciclado ou reutilizado), grandes mudanças e vantagens foram constadas sobre os tratados e coletas desses resíduos comparados aos anos anteriores. Umas delas são: o aumento da vida útil dos aterros sanitários; aumento da conscientização ambiental; geração de energia; melhoria da limpeza da cidade e da qualidade de vida da população; diminuição da poluição do solo, da água e do ar; diminuição de custos de produção pelas indústrias que reaproveitam o material reciclável e a diminuição dos gastos com limpeza urbana (Brasil, 2010).

Dentro do contexto anteriormente descrito, das vantagens da reciclagem ou da coleta seletiva e da importância do tratamento dos resíduos sólidos dentro do cenário nacional, o objetivo deste trabalho é, analisar a viabilidade do aproveitamento energético de gás de aterro na cidade de Juiz de Fora localizada no estado de Minas Gerais, considerando três cenários diferentes usando o programa Waste Reduction Model (WARM).

\section{Material e métodos}

A seguir, são apresentadas as etapas da metodologia adotada e o material usado para realização deste trabalho. Note-se que a metodologia na sua inteira foi baseada em Barros (2013).

\section{Projeção da população e geração de resíduos}

Para a realização deste trabalho, a projeção populacional foi de grande importância para estimar a produção de resíduos sólidos para o município de Juiz de Fora que se localiza no sul do Estado de Minas Gerais, na latitude $21^{\circ} 41^{\prime} 20^{\prime \prime}$ Sul e longitude $43^{\circ} 20^{\prime} 40^{\prime \prime}$ Oeste.

Assim, através dos censos demográficos realizados pelo Instituto Brasileira de Geografia e Estatística (IBGE) nos anos de 1991, 2000 e 2010, foi realizada uma projeção populacional da cidade para um período de 20 anos a partir de 2017 usando o modelo do crescimento logístico, pois este modelo apresenta uma tendência de estabilização dado por um valor máximo da população. 0 histórico da população de Juiz de Fora pode ser observado na Tabela 1.

Tabela 1. Histórico populacional de Juiz de Fora (MG) e condições a serem atendidas para a projeção a partir do modelo do crescimento logístico.

\begin{tabular}{|c|c|c|c|}
\hline \multicolumn{2}{|c|}{ Tempo $\left(t_{n}\right.$ anos $)$} & \multicolumn{2}{|l|}{ População } \\
\hline \multicolumn{2}{|c|}{1991} & \multicolumn{2}{|l|}{385.997} \\
\hline \multicolumn{2}{|c|}{2000} & \multicolumn{2}{|l|}{456.796} \\
\hline \multicolumn{2}{|c|}{2010} & \multicolumn{2}{|l|}{516.247} \\
\hline Condição 1 & $\mathrm{P}_{0}<\mathrm{P}_{1}<\mathrm{P}_{2}$ & $385.997<456.796<516.247$ & Série aceita \\
\hline Condição 2 & $\mathrm{P}_{0} \cdot \mathrm{P}_{2}<\left(\mathrm{P}_{1}\right)^{2}$ & $199.269 .793 .259<208.662 .585 .616$ & Série aceita \\
\hline
\end{tabular}

Fonte: IBGE (2017). 
Onde:

$\mathbf{P}_{\mathbf{0}}$ - População no ano de 1991; $\mathrm{t}_{0}=1991 ;$

$\mathbf{P}_{1}$ - População no ano de 2000; $t_{1}=2000$;

$\mathbf{P}_{2}$ - População no ano de 2010; $\mathrm{t}_{2}=2010$;

Portanto, com esses dados pode-

logístico, que de acordo com Von se calcular o valor da população para Sperling (2005) é obtido através da 2037 usando o modelo de crescimento

Equação 1.

$$
P_{t}=\frac{K_{s}}{1+c \cdot e^{a_{1}\left(t-t_{0}\right)}}
$$

Onde:

$\mathbf{P}_{\mathbf{t}}$ - População estimada do município em 2037;

$\mathbf{K}_{\mathrm{s}}$ - Capacidade de saturação da população;

$\mathbf{a}_{1}$ e c - Coeficientes que controlam a forma e descrevem a localização da curva respectivamente;

$\mathbf{t}_{\mathbf{0}}$ - Refere-se ao ano 1991;

t - Corresponde a cada um dos anos da projeção, no caso de 2017 até 2037.

Ainda segundo Von Sperling (2005), os coeficientes " $K s$ ", " $a_{1}$ " e " $c$ " podem ser determinados, respectiva- mente, através das equações (2), (3) e (4) descritas abaixo.

$$
\begin{gathered}
K_{s}=\frac{2 \cdot P_{0} \cdot P_{1} \cdot P_{2}-P_{1}^{2}\left(P_{0}+P_{2}\right)}{P_{0} \cdot P_{2}-P_{1}^{2}} \\
a_{1}=\frac{1}{\left(t_{2}-t_{1}\right)} \ln \left[\frac{P_{0} \cdot\left(K_{s}-P_{1}\right)}{P_{1} \cdot\left(K_{s}-P_{0}\right)}\right] \\
c=\frac{\left(K_{s}-P_{0}\right)}{P_{0}}
\end{gathered}
$$

\section{Geração do metano e gases de efeito estufa}

A fim de estimar a produção de metano, utilizou-se o programa LandGEM da United States Environmental Protection Agency (USEPA), que por sua vez usa uma equação de primeira ordem para estimar emissões anuais por um período de tempo bem determinado. Conforme a USEPA (2015), para calcular a quantidade de metano a ser gerado, o uso da equação 5 faz-se necessário e também, a adoção dos valores padrões de alguns parâmetros é recomendado.

Assim neste trabalho, foram adotados respectivamente $170\left(\mathrm{~m}^{3} / \mathrm{Mg}\right)$ e $0,05\left(\mathrm{ano}^{-1}\right)$, valores referentes à capacidade do potencial de geração de metano e à taxa de geração de metano respectivamente. 


$$
Q_{C H_{4}}=\sum_{i=1}^{n} \sum_{j=0,1}^{1} k \cdot L_{0} \cdot\left(\frac{M_{i}}{10}\right) \cdot e^{-k \cdot t_{i j}}
$$

Onde:

$\mathbf{C H}_{4}=$ geração anual de metano no ano de cálculo ( $\left.\mathrm{m}^{3} / \mathrm{ano}\right) ;$

$\mathbf{i}=$ incremento de tempo de um ano;

$\mathbf{j}=$ (ano de cálculo) - (ano inicial de recebimento de resíduos);

$\mathbf{n}=$ incremento de tempo de 0,1 ao ano;

$\mathbf{k}=$ taxa de geração de metano $\left(\right.$ ano $\left.^{-1}\right)$;

$\mathbf{L}_{\mathbf{0}}=$ capacidade potencial de geração de metano $\left(\mathrm{m}^{3} / \mathrm{Mg}\right) ;$

$\mathbf{M i}=$ massa de resíduos aceita no enésimo ano $(\mathrm{Mg})$;

$\mathbf{t}_{\mathbf{i j}}=$ idade da j-ésima seção de massa de resíduo aceita no enésimo ano.

Já para a projeção dos gases de efeito estufa, foi utilizado para a realização deste trabalho o programa Waste Reduction Model (WARM) da USEPA. 0 principal objetivo deste programa é auxiliar organizações e projetos que estão relacionados a resíduos sólidos a reduzir os gases de efeito estufa por meio de práticas de gestão de resíduos (USEPA, 2015).

Tabela 2. Projeção populacional e geração de resíduos para os próximos 20 anos para a cidade de Juiz de Fora (MG).

\begin{tabular}{|c|c|c|c|c|c|c|}
\hline Ano & População & $\begin{array}{l}\text { Índice per } \\
\text { capita de } \\
\text { resíduos } \\
\text { sólidos, com } \\
\text { acréscimo de } \\
\text { 1\% (kg/hab.d) }\end{array}$ & $\begin{array}{l}\text { Quantidade } \\
\text { diária } \\
\text { coletada de } \\
\text { resíduos } \\
\text { sólidos } \\
\text { urbanos } \\
\text { (kg/d) }\end{array}$ & $\begin{array}{l}\text { Quantidade } \\
\text { anual } \\
\text { coletada de } \\
\text { resíduos } \\
\text { sólidos } \\
\text { urbanos } \\
\text { (kg/ano) }\end{array}$ & $\begin{array}{l}\text { Quantidade } \\
\text { anual } \\
\text { coletada de } \\
\text { resíduos } \\
\text { sólidos } \\
\text { urbanos } \\
\text { (t/ano) }\end{array}$ & $\begin{array}{c}\text { Quantidade } \\
\text { anual } \\
\text { acumulada } \\
\text { coletada de } \\
\text { resíduos sólidos } \\
\text { urbanos (t/ano) }\end{array}$ \\
\hline 2017 & 545429 & 0,935 & 510202 & 186223763 & 186224 & 186224 \\
\hline 2018 & 549816 & 0,945 & 519449 & 189598922 & 189599 & 375823 \\
\hline 2019 & 554070 & 0,954 & 528703 & 192976577 & 192977 & 568799 \\
\hline 2020 & 558193 & 0,964 & 537963 & 196356530 & 196357 & 765156 \\
\hline 2021 & 562185 & 0,973 & 547229 & 199738651 & 199739 & 964894 \\
\hline 2022 & 566050 & 0,983 & 556501 & 203122870 & 203123 & 1168017 \\
\hline 2023 & 569789 & 0,993 & 565779 & 206509182 & 206509 & 1374526 \\
\hline 2024 & 573404 & 1,003 & 575062 & 209897637 & 209898 & 1584424 \\
\hline 2025 & 576898 & 1,013 & 584352 & 213288342 & 213288 & 1797712 \\
\hline 2026 & 580273 & 1,023 & 593648 & 216681456 & 216681 & 2014394 \\
\hline 2027 & 583531 & 1,033 & 602951 & 220077187 & 220077 & 2234471 \\
\hline 2028 & 586676 & 1,044 & 612262 & 223475790 & 223476 & 2457947 \\
\hline 2029 & 589709 & 1,054 & 621582 & 226877564 & 226878 & 2684824 \\
\hline 2030 & 592634 & 1,065 & 630912 & 230282846 & 230283 & 2915107 \\
\hline 2031 & 595453 & 1,075 & 640262 & 233692016 & 233692 & 3148799 \\
\hline 2032 & 598169 & 1,086 & 649604 & 237105484 & 237105 & 3385905 \\
\hline 2033 & 600785 & 1,097 & 658969 & 240523696 & 240524 & 3626429 \\
\hline 2034 & 603303 & 1,108 & 668348 & 243947127 & 243947 & 3870376 \\
\hline 2035 & 605726 & 1,119 & 677743 & 247376278 & 247376 & 4117752 \\
\hline 2036 & 608057 & 1,130 & 687155 & 250811679 & 250812 & 4368564 \\
\hline 2037 & 610299 & 1,141 & 696586 & 254253879 & 254254 & 4622817 \\
\hline
\end{tabular}


Ainda de acordo com a USEPA (2015), além de calcular as emissões de gases de efeito estufa de cenários base, o programa calcula também as emissões em toneladas métricas tanto de carbono equivalente (MTCE) quanto de dióxido de carbono equivalente $\left(\mathrm{MTCO}_{2} \mathrm{E}\right)$, e unidades de energia (milhões de BTU) por uma diversa variedade de tipos de materiais que são encontrados nos resíduos sólidos urbanos.

\section{Resultados e discussão}

Os valores da projeção populacional obtida a cada ano, de 2017 até 2037 e também da geração de resíduos durante o mesmo período estão apresentados na Tabela 2.

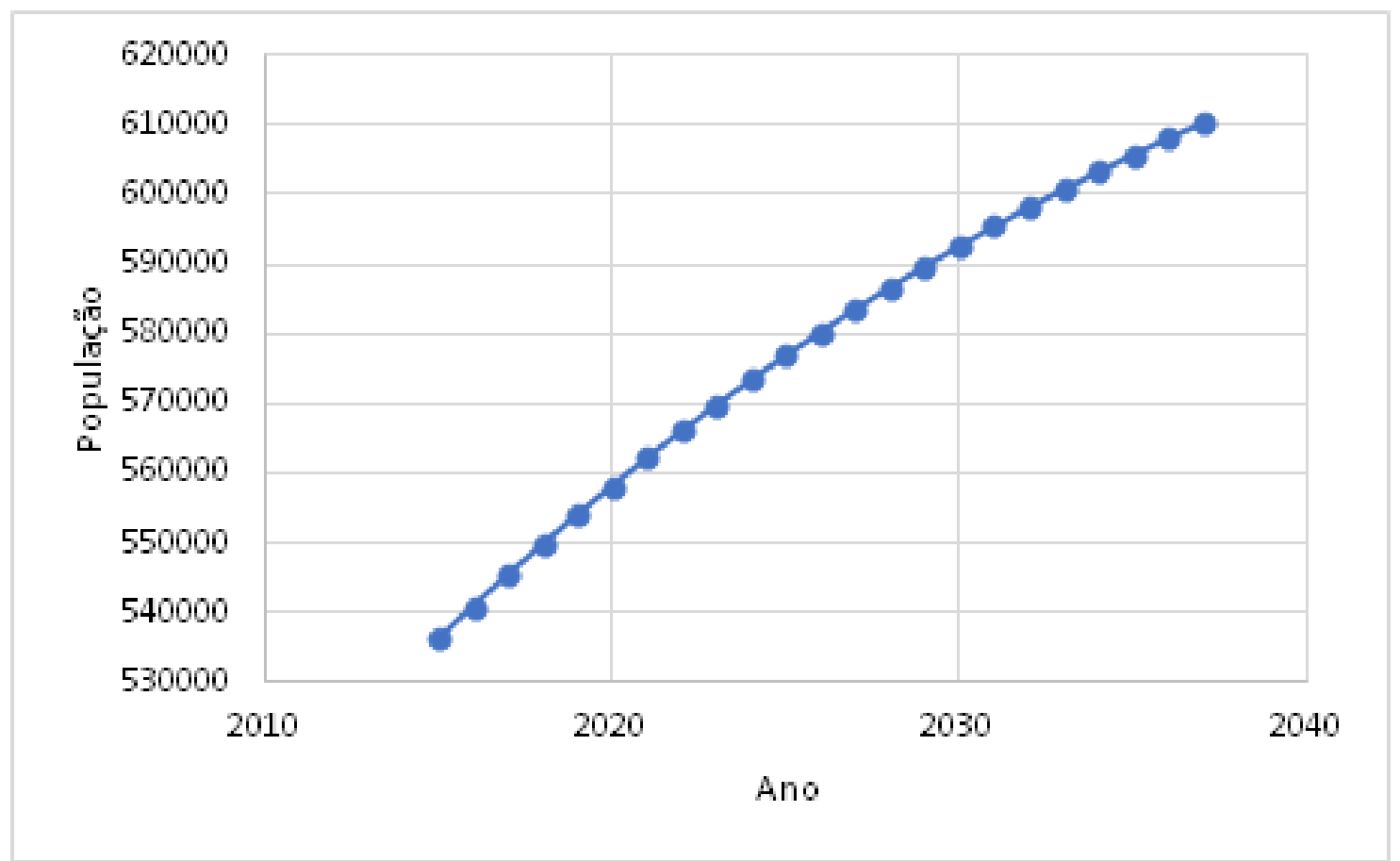

Figura 1. Crescimento populacional de Juiz de Fora (MG) até o ano de 2037.

Projeção populacional para a Cidade de Juiz de Fora (MG)

A Figura 1 a seguir apresenta o comportamento do crescimento da população da cidade de Juiz de Fora ao longo dos anos.

De acordo com o modelo de projeção usado neste trabalho, a saturação do crescimento populacional para a cidade de estudo seria atingida no ano de 2282, sendo um aumento de
116.065 pessoas em relação ao ano de 2017.

Projeção da geração de resíduos sólidos urbanos para a cidade de Juiz de Fora (MG)

Foi possível obter também a partir dos cálculos uma projeção da quantidade anual coletada de resíduos sólidos urbanos no decorrer dos anos, conforme a Figura 2. 


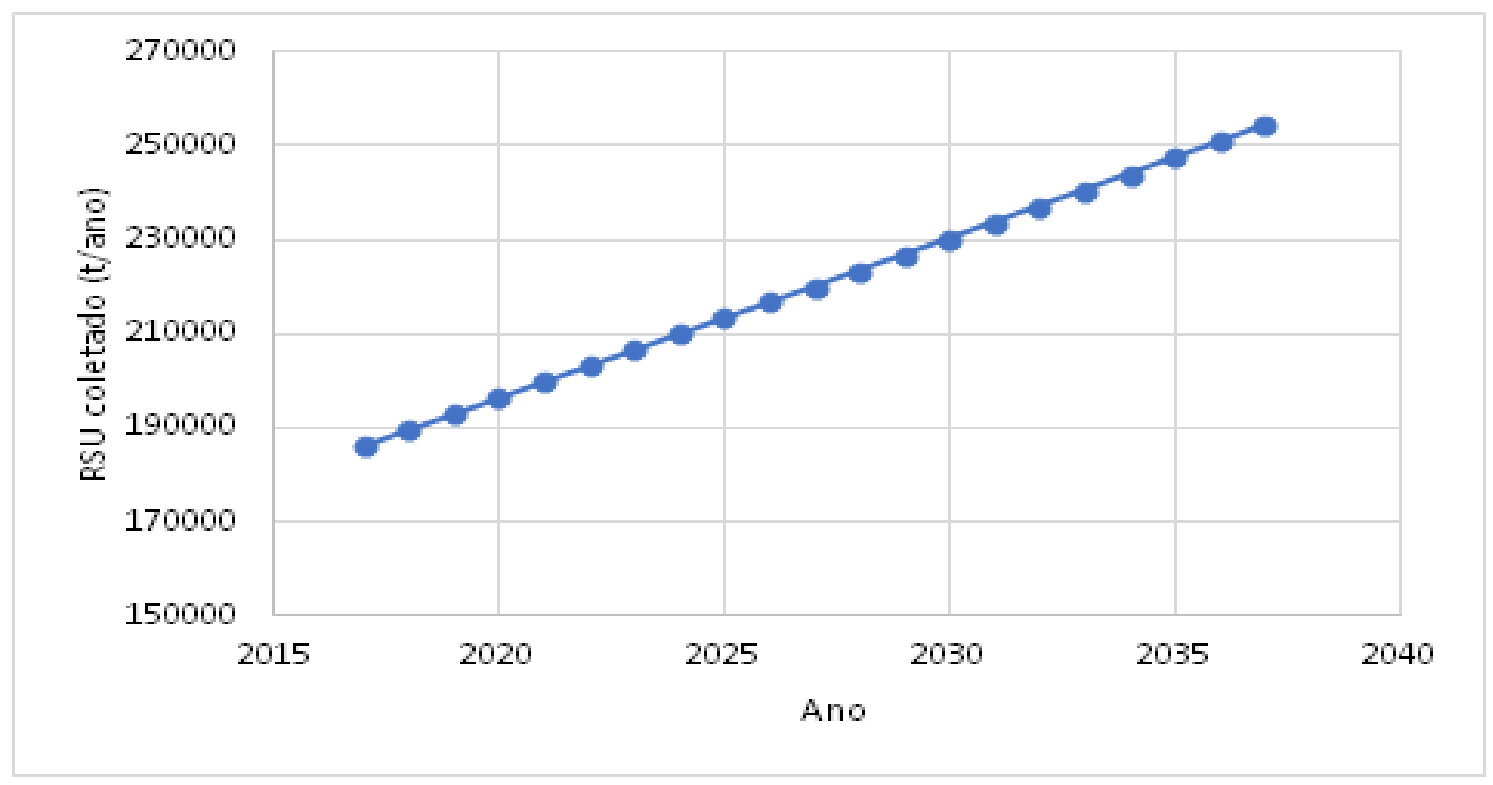

Figura 2. Quantidade anual coletada de resíduos sólidos urbanos, em toneladas em Juiz de Fora (MG).

Da análise da Figura 2, observe-se que a medida que os anos passam, a quantidade anual de geração de resíduos sólidos urbanos aumenta de maneira linear. Isto quer dizer que esse aumento será contínuo até o ano de saturação da população. Segundo a projeção realizada, o ano de 2037 tem uma quantidade de resíduos coletadas aproximadamente igual a 11\% maior que em 2017.
Portanto, pode concluir-se que o aumento da geração de resíduos acompanha o crescimento populacional da cidade de estudo.

\section{Geração de metano}

As Figuras 3 e 4 a seguirem apresentam a projeção da produção de gases no aterro sanitário na cidade de Juiz de Fora (MG).

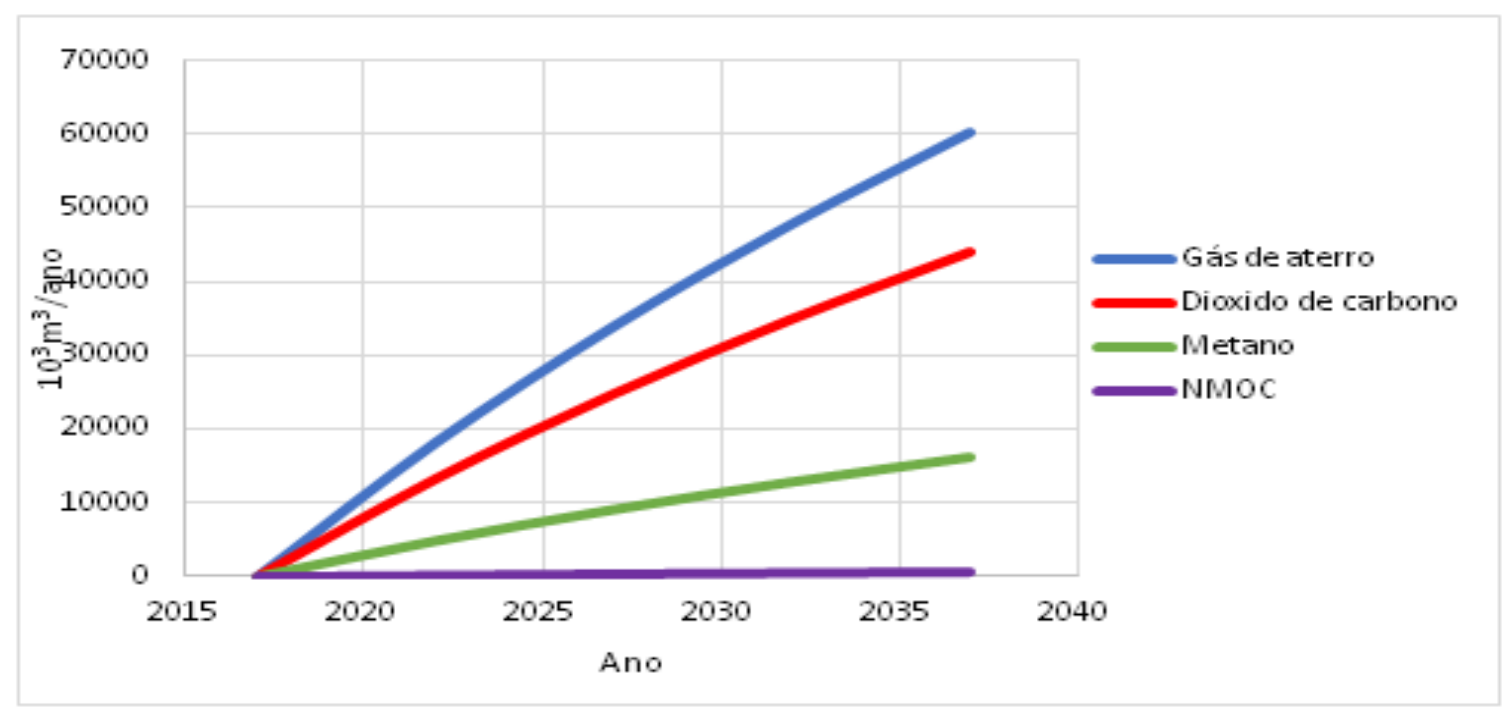

Figura 3. Produção de gases no aterro sanitário de Juiz de Fora (MG) até 2040. 


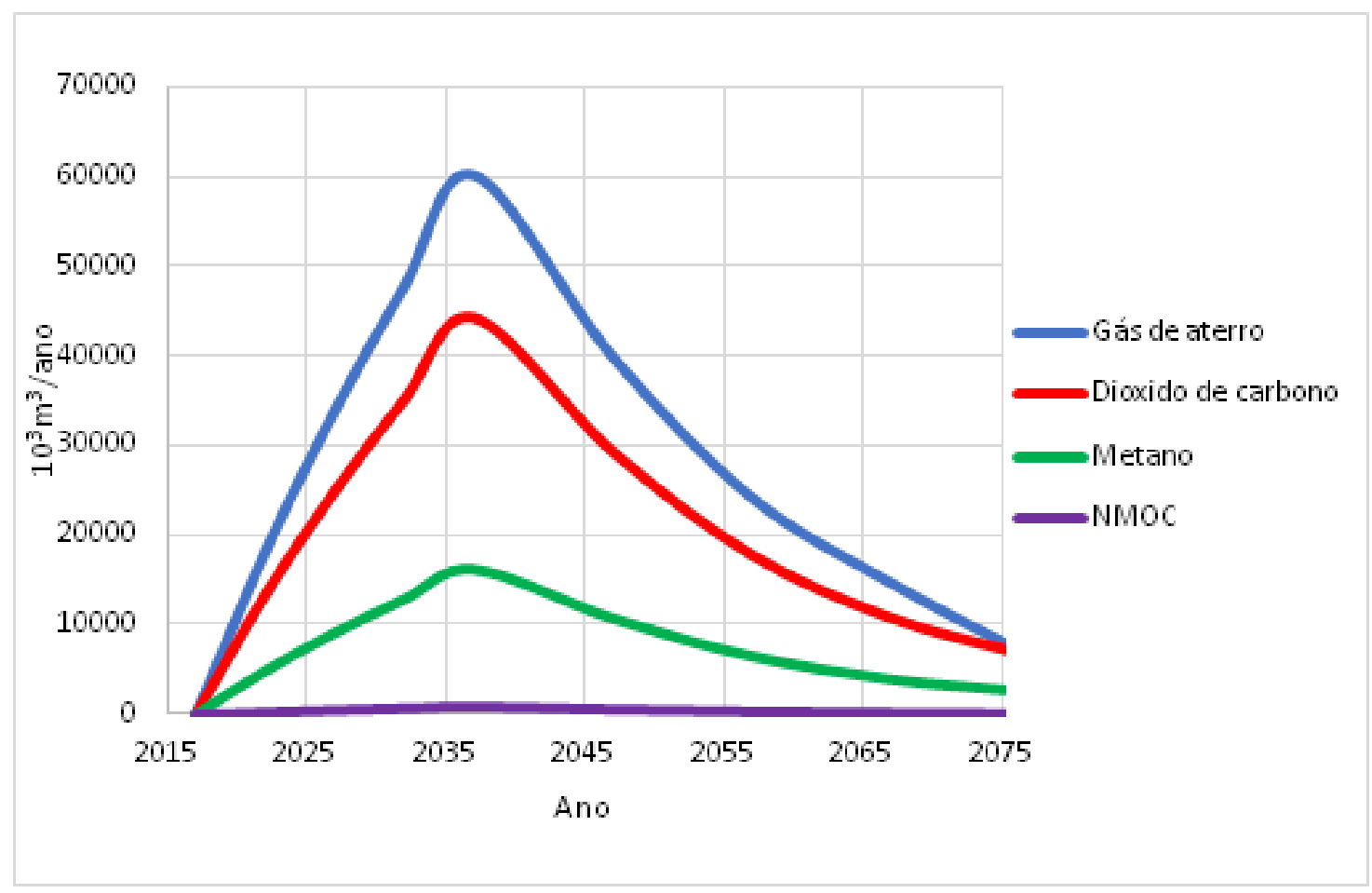

Figure 4. Produção de gases no aterro sanitário de Juiz de Fora (MG) até 2075.

De acordo com os gráficos acima, percebe-se que a produção de gases atinge seu pico no ano de 2037, ou seja, no último ano do encerramento do projeto, gerando um valor total de $60,268 \times 10^{6} \mathrm{~m}^{3}$ de gás de aterro, dos quais $16,098 \times 10^{6} \mathrm{~m}^{3}$ são metano, considerado como o gás de maior interesse para conversão energética. estufa

\section{Geração de gases de efeito}

Para análise da geração de gases de efeito estufa em relação ao aterro sanitário de Juiz de Fora, foi considerada a quantidade de resíduos sólidos gerados no ano de 2017, que através dos cálculos realizados deu um valor igual a 186.224 toneladas.

A composição gravimétrica comsiderada neste presente trabalho foi a constante no Diagnóstico dos Resíduos Sólidos Urbanos de 2012, do Departamento Municipal de Limpeza Urbana (DEMLURB, 2012). A Tabela 3 a seguir, mostra estes valores.

Tabela 3. Composição gravimétrica e quantidade de resíduos para o município de Juiz de Fora (MG).

\begin{tabular}{|l|c|c|}
\hline Material & Porcentagem (\%) & Quantidade (T) \\
\hline Metais & 3,68 & 6853,04 \\
\hline Papel, papelão & 17,47 & 32533,33 \\
\hline Plástico & 14,10 & 26257,58 \\
\hline Vidro & 1,62 & 3016,82 \\
\hline Matéria orgânica & 58,73 & 109369,35 \\
\hline Pano, trapo & 4,40 & 8193,85 \\
\hline Total & $\mathbf{1 0 0}$ & $\mathbf{1 8 6 2 2 4}$ \\
\hline
\end{tabular}

Fonte: Departamento Municipal de Limpeza Urbana (DEMLURB, 2012). 
Assim neste trabalho, foram considerados três cenários usando o programa Waste Reduction Model (WARM) da USEPA. Um dos cenários é o de base, que considera a hipótese de que todos os materiais vão diretamente para o aterro sanitário, sem reciclagem, nem redução, nem compostagem ou incineração. Este cenário de base por sua vez, é dividido em dois cenários $\mathrm{A}$ e $\mathrm{B}$, onde o cenário de base $\mathrm{A}$ não leva em consideração a recuperação de gás de aterro, enquanto que o cenário de base $B$ leva em consideração tanto a recuperação de gás de aterro quanto a conversão energética.

Foi feita uma comparação entre o cenário de base $\mathrm{A}$ e o cenário 1 , que considerou percentagens de materiais reciclados baseados em dados do DEMLURB (2012). Considerou-se para os metais, uma taxa de reciclagem igual a $35 \%$; para papel/papelão $45 \%$ e para os plásticos e vidros uma taxe $20 \%$ respectivamente. Os resultados obtidos estão apresentados na Tabela 4.

Tabela 4. Resultados da simulação com o WARM para o cenário de base "A" e cenário 1.

\begin{tabular}{|l|c|c|c|}
\hline Tipo de cenário & $\begin{array}{c}\text { Emissões totais } \\
\text { de GEE (MTCE) }\end{array}$ & $\begin{array}{c}\text { Emissões totais } \\
\text { de GEE (MTCO } \mathbf{2} \text { E) }\end{array}$ & $\begin{array}{c}\text { Alteração total no uso } \\
\text { energético (milhões de BTU) }\end{array}$ \\
\hline Cenário de base A & 163826 & 600695 & 7.279 .763 \\
\hline Cenário 1 & 45898 & 168291 & -2.170 .191 \\
\hline
\end{tabular}

Da análise da Tabela 4 , percebese que mesmo com a ausência de coleta do gás de aterro neste caso, a reciclagem dos materiais traz muitos benefícios para a cidade como por exemplo a redução das emissões de gases do efeito estufa.

Também, foi feito um estudo comparativo entre o cenário de base $\mathrm{B}$ e os cenários 2 e 3 . No caso do cenário 2, foram adotadas as mesmas percentagens de reciclados mencionadas acima, ou seja, são baseados em valores do DEMLURB (2012). Já no cenário 3, considerado como cenário otimista, considerou-se uma taxa de redução na fonte de vidro de $20 \%$, passando para $40 \%$ com reciclagem; para $65 \%$ na reciclagem de papéis; $55 \%$ para o que é de metais; $40 \%$ para os plásticos e $20 \%$ para a compostagem da matéria orgânica. A Tabela 5 apresenta os resultados obtidos por cenário 2 e 3 .

Tabela 5. Resultados da simulação com o WARM para o cenário de base "B" e cenários 2 e 3.

\begin{tabular}{|c|c|c|c|}
\hline Tipo de cenário & $\begin{array}{c}\text { Emissões totais } \\
\text { de GEE (MTCE) }\end{array}$ & $\begin{array}{c}\text { Emissões totais de } \\
\text { GEE } \text { (MTCO }_{\mathbf{2}} \text { E) }\end{array}$ & $\begin{array}{c}\text { Alteração total no uso } \\
\text { energético (milhões de BTU) }\end{array}$ \\
\hline Cenário de base B & -28192 & -103370 & -6.934 .859 \\
\hline Cenário 2 & -89737 & -329034 & -7.239 .441 \\
\hline Cenário 3 & -117081 & -429298 & -11.570 .734 \\
\hline
\end{tabular}

Comparando os cenários de base A e B, percebe-se que o valor obtido no cenário B é menor que aquele obtido no cenário A. Isso pode ser explicado pelo fato que o cenário de base A não levou em consideração a recuperação de gás de aterro. Portanto, a recuperação de gás de aterro torne-se um fator importante que 
deve ser considerado já que o gás recuperado poderá ser utilizado para conversão energética. Note-se também, uma grande diminuição de emissões de gases de efeito estufa quando se compara os cenários 2 e 3 apresentados na Tabela 5 acima. Isto mostra quanto são importantes a redução da produção de resíduos e o aumento da reciclagem de materiais.

\section{Análise da viabilidade econômica}

A análise de viabilidade econômica é um tipo de análise que tem como objetivo medir a sensibilidade dos indicadores de viabilidade a determinadas variáveis fundamentais da análise e assim, analisar o grau de incerteza e de risco das conclusões obtidas. De fato, qualquer estudo de viabilidade econômica e financeira inclui sempre algum grau de incerteza o qual, apesar de poder ser reduzido através de detalhados estudos de mercado, nunca deixa de existir (Nunes, 2015).

Assim sendo, no presente trabalho a análise da viabilidade econômica foi feita considerando um tempo de 20 anos para o empreendimento e uma taxa de juros igual a 8,5\%/ano. Os cálculos de Valor Presente Líquido (VPL) e da Taxa Interna de Retorno (TIR) foram realizados em planilha eletrônica conforme as equações 6 e 7.

$$
\begin{aligned}
& \mathrm{VPL}=\sum_{t=1}^{n} \frac{F C}{(1+i)^{t}}-\mathrm{I} \\
& \mathrm{TIR}=\sum_{t=1}^{n} \frac{F C}{\left(1+i^{*}\right)^{t}}-\mathrm{I}
\end{aligned}
$$

Onde:

$\mathbf{F C}=$ Fluxo de caixa $(\mathrm{R} \$)$

$\mathbf{i}=$ Taxa de desconto $(\%)$;

$i^{*}=$ Taxa de interesse (\%);

$\mathbf{I}=$ Investimento $(\mathrm{R} \$)$;

$\mathbf{n}=$ Número máximo de tempo (ano);

$\mathbf{t}=$ Número de tempo (ano).

Os resultados obtidos deram respectivamente $R \$ 1.039 .314,27$ e 9,8\%, com um investimento inicial igual a $\mathrm{R} \$$ 12.419.370,06 considerando uma potência de 0,99 MW a ser gerada anualmente. Isto quer dizer que o projeto é viável, pois o valor da taxa interna de retorno obtido é maior do que a taxa inicial (TMA), que foi de $8,5 \%$, mas não tão atrativo devido às questões ambientais.

Note-se também que os cálculos da análise da viabilidade foram feitos considerando cada cenário apresentado acima e percebeu-se que aquele que se apresentou economicamente viável com um maior valor de VPL entres os três cenários foi o primeiro cenário, sem aproveitamento de energia.

\section{Conclusão}

A Cidade de Juiz de Fora representa o quarto município mais populoso do estado de Minas Gerais segundo o Instituto Brasileiro de Geografia e Estatística (IBGE), e se encontra em uma fase de crescimento econômico e populacional constante. Uma das consequências desse crescimento é o aumento na geração de resíduos sólidos, sejam eles domésticos ou industriais. 0 tratamento de toda essa geração de resíduos é de extrema 
importância para que não haja depreciação nos recursos naturais da região, o que implicaria em diversos problemas ambientais e de saúde pública, sendo assim, a coleta seletiva é fundamental para a população.

A fim de garantir uma melhor qualidade de vida para a população e as gerações futuras, o presente trabalho objetivou a avaliar uma série de fatores relacionados aos resíduos sólidos gerados na cidade de estudo. Para isso, realizou-se primeiramente uma projeção populacional para 2037 usando o modelo de crescimento logístico, que resultou em uma população total de 610299 habitantes com uma população de saturação que será atingida no ano de 2282, sendo um aumento de 116065 pessoas em relação ao ano de 2017. Enquanto isso, a geração dos resíduos sólidos permanecerá crescente, passando de 186224 toneladas em 2017 para 254254 toneladas em 2037.

Os resultados da projeção da geração de gás proveniente do aterro sanitário mostraram que o pico da produção de gás será atingido em 2037, justamente no ano do encerramento do projeto com um valor total de $60,268 \mathrm{x}$ $10^{6} \mathrm{~m}^{3}$, dos quais $16,098 \times 10^{6} \mathrm{~m}^{3}$ são metano, considerado como o gás de maior interesse para conversão energética. Enfim, com o auxílio do programa WARM, foi possível avaliar três cenários diferentes que através de um estudo de viabilidade econômica possibilitou a identificação do melhor cenário de aproveitamento energético, porém não atrativo para a cidade.

\section{Conflito de interesses}

Os autores declaram não haver conflito de interesses.

\section{Referências}

ABNT - Associação Brasileira de Normas Técnicas. NBR 10.004 - Resíduos Sólidos Classificação. Rio de Janeiro: ABNT, 2004.

Barros, R. M. Tratado sobre resíduos sólidos: gestão, uso e sustentabilidade. 1 . ed. Rio de Janeiro: Interciência, 2013.

Brasil. Lei no 12.305, de 2 de agosto de 2010. Institui a Política Nacional de Resíduos Sólidos; altera a Lei $\mathrm{n}$ o 9.605, de 12 de fevereiro de 1998; e dá outras providências. Disponível em: <http://www.planalto. gov.br/ccivil_03/_ato2007-2010/2010/lei/ 112305.htm>. Acesso em: 20 ago. 2018.

DEMLURB - Departamento Municipal de Limpeza Urbana. 2012. Disponível em: <http://www.demlurb.pjf.mg.gov.br/>. Acesso em: 20 ago. 2018.

IBGE - Instituto Brasileiro de Geografia e Estatística. Infográficos: Evolução populacional e pirâmide etária da cidade de Juiz de Fora (MG). Disponível em: <https://cidades. ibge.gov.br/painel/painel.php?lang=\&codmu $n=313670 \&$ search=minas-gerais $\mid$ juiz-defora|infograficos:-dados-gerais-domunicipio>. Acesso em: 13 ago. 2018.

Mansor, M. T. C.; Camarão, T. C. R. C.; Capelini, M.; Kovacs, A.; Filet, M.; Santos, G. A.; Silva, A. B. Resíduos sólidos. São Paulo: Governo do Estado de São Paulo, Secretaria do Meio Ambiente, 2010. (Série Cadernos de Educação Ambiental, 6).

Nunes, P. Conceito de análise de sensibilidade. 2015. Disponível em: <http://knoow.net/cienceconempr/gestao/a nalise-de-sensibilidade>. Acesso em: 20 ago. 2018.

USEPA - United States Environmental Protection Agency. Waste Reduction Model (WARM). 2015. Disponível em: <http://www3.epa.gov/warm/>. Acesso em: 21 ago. 2018.

USEPA - United States Environmental Protection Agency. Landfill gas emission model. Version 3.02. User's guide. 2015. Disponível em: <http://www3.epa.gov/ ttncatc1/dir1/landgem-v302-guide.pdf>.

Acesso em: 21 ago. 2018. 
Von Sperling, M. Introdução à qualidade das águas e ao tratamento de esgotos. 2. ed. rev. Belo Horizonte: Universidade Federal de Minas Gerais, Departamento de Engenharia Sanitária e Ambiental, 2005. v. 1. seja devidamente citada. 